\title{
GLOBAL DYNAMICS OF THE HOŘAVA-LIFSHITZ COSMOLOGICAL SYSTEM
}

\author{
FABAO GAO ${ }^{1,2}$, AND JAUME LLIBRE ${ }^{2}$
}

ABSTRACT. Using the qualitative theory, the global dynamics of the cosmological model based on Hořava-Lifshitz gravity is described.

\section{INTRODUCTION}

Ten years ago Hořava [1] put forward a theory of spacetime asymmetric gravity, which is similar to Lifshitz's scalar field theory. If the spatial dimension in Lifshitz's scalar field theory has a weight of one, then the time dimension has a weight of three. Therefore this theory is also known as Hořava-Lifshitz gravity. It ignited a great deal of research on the possible application of this theory in cosmology and black hole physics (see [2]-[5] or the review articles [6], [7] and the references therein).

With or without detailed-balance conditions, Leon and Saridakis [8] carried out a detailed phase space analysis of Hořava-Lifshitz cosmology, and found that the universe governed by Hořava gravity had latetime solutions compatible with observations. They also presented several results on the stability of de Sitter solutions in Hořava-Lifshitz cosmology by using the central manifold theory [9]. Furthermore Saridakis [10] reviewed some general aspects of Hořava-Lifshitz cosmology and extracted cosmological equations from its basic version. He proved Key words and phrases. Hořava-Lifshitz, Global dynamics, cosmology. 
that bouncing and cycling can occur naturally by phase space analysis. However, he also showed that Hořava-Lifshitz gravity is affected by instability in its basic version with detailed perturbation analysis. For the stability of gravitational scalar mode Chen [11] briefly summarized the Hořava-Lifshitz theory of gravity and its modifications and its implications in cosmology.

In recent years Lepe and Saavedra [12] discussed some aspects of Hořava-Lifshitz cosmology with emphasis on some cosmological solutions that exist in general relativity (Friedmann cosmology), especially in the flat case that dust-driven evolution is the same in both cosmological theories. For Hořava-Lifshitz theory of gravitation Abreu et al. [13] explored a non-commutative version of the FriedmannRobertson-Walker cosmological model, in which material content is described by ideal fluids, and the constant curvature of the spatial sections can be positive, negative or zero. Under the background spacetime of Friedmann-Lemaître-Robertson-Walker, Paliathanasis and Leon [14] divided the integrability of Hořava-Lifshitz scalar field into four cases according to the existence of cosmological constant term and the disappearance of space curvature. They followed the singularity analysis method to determine integrability. It is believed that their work will be very helpful to the integrability of the gravitational field equation in cosmology. In addition to the cosmological solutions in [14], two versions of Hořava-Lifshitz gravity were discussed for finding and analyzing the plane symmetric, static (non-static) solutions in HořavaLifshitz gravity [15]. They also studied the plane symmetry of the new modified version of Hořava gravity. 
Here we shall describe the global dynamics of the Hořava-Lifshitz cosmological model in a Friedmann-Lemaître-Robertson-Walker spacetime with zero curvature and without the cosmological constant term. This dynamics is provided by the gravitational field equations in dimensionless variables given by

$$
\begin{aligned}
\frac{d y_{1}}{d t} & =\left(y_{1}^{2}-1\right)\left(3 y_{1}-\sqrt{6} y_{3}\right) \\
\frac{d y_{2}}{d t} & =\left(3 y_{1}^{2}-2\right) y_{2} \\
\frac{d y_{3}}{d t} & =-2 \sqrt{6} y_{1} f\left(y_{3}\right)
\end{aligned}
$$

with the power law potential $f\left(y_{3}\right)=-y_{3}^{2} /(2 n)$, where $n$ is a natural number. See equations (17)-(19) of [14] for more details.

The description of dynamics of system (1) is given in section 4 .

\section{Phase Portraits on the InVARIANt Planes}

In order to study the phase portraits of system (1), we start studying the phase portraits of its invariant planes

$$
y_{1}= \pm 1, y_{2}=0, y_{3}=0
$$

After we will study the local phase portraits of the finite and infinite equilibrium points, and finally the global phase portraits in the region $-1 \leq y_{1} \leq 1$, which is the interest region for cosmology.

2.1. The invariant plane $y_{1}=1$. On this plane system (1) becomes

$$
\frac{d y_{2}}{d t}=y_{2}, \frac{d y_{3}}{d t}=\frac{\sqrt{6}}{n} y_{3}^{2} .
$$


The unique finite equilibrium point is $q_{y_{1,1}}=(0,0)$. It is a semihyperbolic equilibrium point and using Theorem 2.19 of [16] $q_{y_{1,1}}$ is a saddle-node.

Based on the Poincaré transformation $y_{2}=1 / v, y_{3}=u / v$, on the local chart $U_{1}$ (see for more details on the Poincaré compactification Chapter 5 of [16]) equations (2) become

$$
\dot{u}=\frac{\sqrt{6}}{n} u^{2}-u v, \dot{v}=-v .
$$

This system has the infinite semi-hyperbolic equilibrium point $p_{y_{1,1}}=$ $(0,0)$. Applying to it Theorem 2.19 of [16] we obtain the saddle-node shown in Figure 1(a).

Similarly on the local chart $U_{2}$ system (2) is

$$
\dot{u}=-\frac{\sqrt{6}}{n} u+u v, \dot{v}=-\frac{\sqrt{6}}{n} v
$$

Then the equilibrium point $p_{y_{1,2}}=(0,0)$ of system (4) is a hyperbolic stable node with eigenvalues $-\sqrt{6} / n$ of multiplicity two.

Finally joining the previous information on the studied three equilibrium points $q_{y_{1,1}}, p_{y_{1,1}}$ and $p_{y_{1,2}}$, together with the diametrically opposite equilibrium points $P_{y_{1,1}}$ and $P_{y_{1,2}}$ at infinity of $p_{y_{1,1}}$ and $p_{y_{1,2}}$, we obtain the global phase portrait of system (2) in the Poincaré disc of the invariant plane $y_{1}=1$ in Figure 1(b). Here we have also used that the straight lines $y_{2}=0$ and $y_{3}=0$ are invariant by the flow of system (2). 


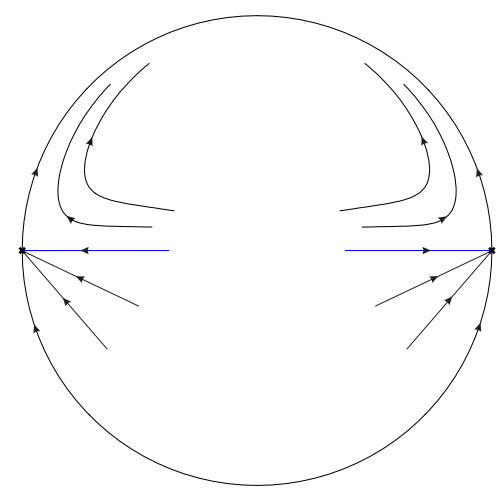

(a)

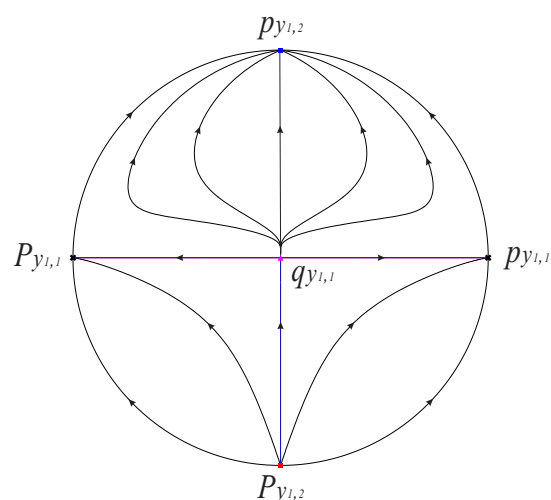

(b)

Figure 1. In (a) there is the local phase portraits of the saddle-node $p_{y_{1,1}}=(0,0)$ in the Poincaré disc. In (b) there is the phase portraits of the invariant plane $y_{1}=1$.

2.2. The invariant plane $y_{1}=-1$. On this plane system (1) reduces to

$$
\frac{d y_{2}}{d t}=y_{2}, \frac{d y_{3}}{d t}=-\frac{\sqrt{6}}{n} y_{3}^{2}
$$

System (5) is similar to system (2), so the equilibrium point $q_{y_{1,2}}=$ $(0,0)$ is a saddle-node.

On the local chart $U_{1}$ system (5) is

$$
\dot{u}=-\frac{\sqrt{6}}{n} u^{2}-u v, \dot{v}=-v .
$$

The semi-hyperbolic equilibrium point $p_{y_{1,3}}=(0,0)$ is a saddle-node by Theorem 2.19 of [16], and its local phase portrait in the Poincaré disc is described in Figure 2(a).

On the local chart $U_{2}$ system (5) becomes

$$
\dot{u}=\frac{\sqrt{6}}{n} u+u v, \dot{v}=\frac{\sqrt{6}}{n} v .
$$




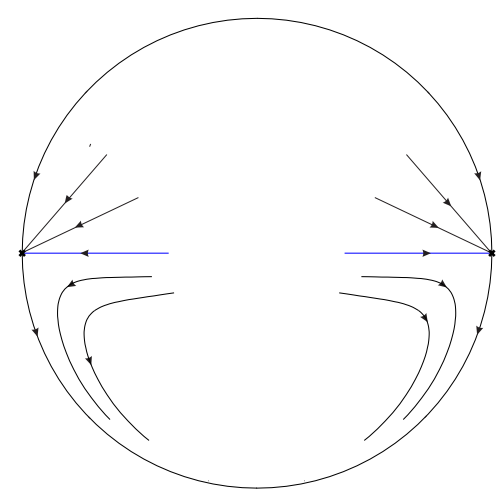

(a)

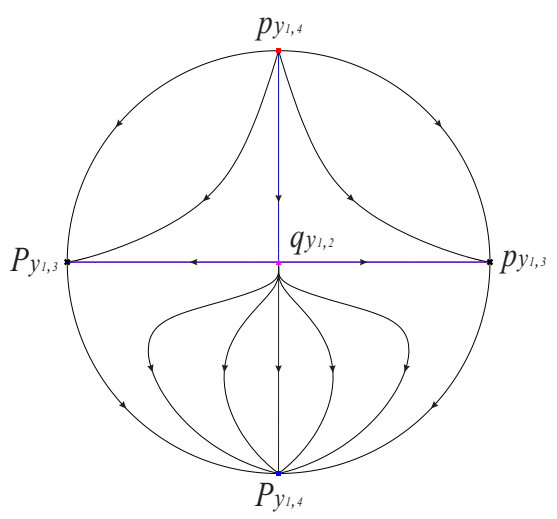

(b)

Figure 2. In (a) there is the local phase portraits of the saddle-node $p_{y_{1,3}}=(0,0)$ in the Poincaré disc. In (b) there is the phase portraits of the invariant plane $y_{1}=-1$.

Its equilibrium point $p_{y_{1,4}}=(0,0)$ is a hyperbolic unstable node with eigenvalue $\sqrt{6} / n$ of multiplicity two.

In short joining the previous information as we did for the plane $y_{1}=1$, we get the global phase portraits in the Poincaré disc of the invariant plane $y_{1}=-1$ in Figure 2(b). Furthermore by using the method of Poincaré compactification $y_{1}=z_{1} / z_{3}, y_{2}=1 / z_{3}, y_{3}=z_{2} / z_{3}$ and the change of time $d t=z_{3}^{3} d \tau$ in $\mathbb{R}^{3}$, then the analytical vector field of system (1) in the local chart $U_{2}$ becomes

$$
\begin{aligned}
z_{1}^{\prime} & =-\sqrt{6} z_{1}^{2} z_{2}-z_{1} z_{3}^{2}+\sqrt{6} z_{2} z_{3}^{2}, \\
z_{2}^{\prime} & =z_{2}\left(-3 z_{1}^{2}+\frac{\sqrt{6}}{n} z_{1} z_{2}+2 z_{3}^{2}\right)\left(-1+z_{3}^{2}\right), \\
z_{3}^{\prime} & =-3 z_{1}^{2} z_{3}+2 z_{3}^{3},
\end{aligned}
$$

where the prime denotes derivative with respect to the time $\tau$. Then this system has the equilibrium points $\left(z_{1}, z_{2}, z_{3}\right)=(0, a, 0)$ for all $a$. So the infinity of Figure 3 is filled up with equilibrium points when $y_{1}=0$. 
Therefore combining Figures 1(b) and 2(b) we obtain the phase portrait of system (1) inside the Poincaré ball as shown in Figure 3. All the points on the dashed circle in the plane $y_{1}=0$ are the equilibrium points.

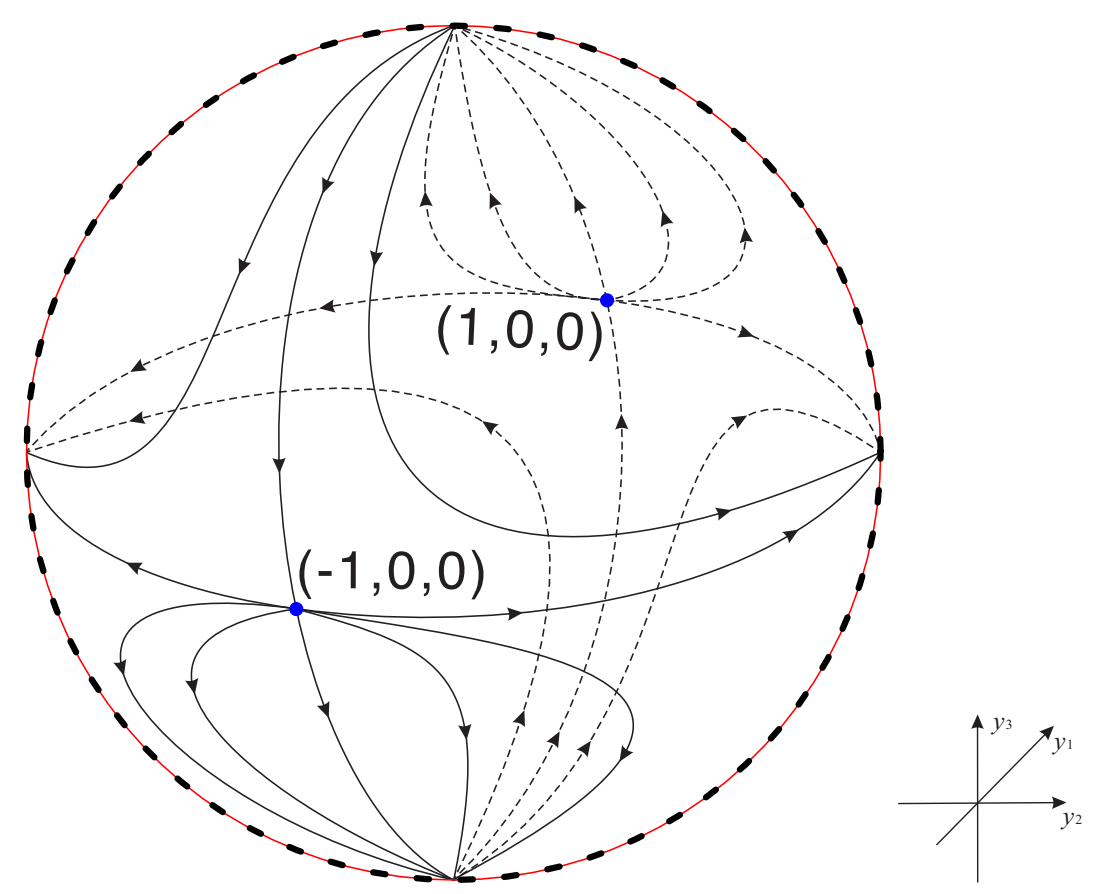

Figure 3. Phase portraits on the planes $y_{1}= \pm 1$ inside the Poincaré ball.

2.3. The invariant plane $y_{2}=0$. On this plane system (1) can be rewritten as

$$
\frac{d y_{1}}{d t}=\left(y_{1}^{2}-1\right)\left(3 y_{1}-\sqrt{6} y_{3}\right), \frac{d y_{3}}{d t}=\frac{\sqrt{6}}{n} y_{1} y_{3}^{2} .
$$

This system has three equilibrium points $O_{y_{2}, 0}=(0,0), q_{y_{2,1}}=(1,0)$, $q_{y_{2,2}}=(-1,0)$, which are semi-hyperbolic.

In order to apply Theorem 2.19 of [16], the linear part at each equilibrium point of system (9) must be written into its real Jordan normal 
form. So we do the following transformation to the linear part of $O_{y_{2}, 0}$

$$
y_{1}=\frac{\sqrt{6}}{3}(y-x), \quad y_{3}=-x
$$

Then equations (9) become

$$
\begin{aligned}
& \dot{x}=-\frac{2}{n} x^{3}+\frac{2}{n} x^{2} y, \\
& \dot{y}=3 y-\frac{2}{n} x^{3}+\frac{1}{2}\left(\frac{1}{n}-1\right) x^{2} y+4 x y^{2}-2 y^{3} .
\end{aligned}
$$

Let $y=g(x)=2 x^{3} / 3 n+4(n-1) x^{5} /\left(9 n^{2}\right)+\mathcal{O}\left(x^{5}\right)$ be the solution of the second equation of system (10). Substituting $y=g(x)$ into the first equation of system (10), we obtain $\dot{x}=-2 x^{3} / n+\mathcal{O}\left(x^{5}\right)$. So by Theorem 2.19 of [16], $O_{y_{2}, 0}=(0,0)$ is a saddle point.

Applying again Theorem 2.19 of [16] to the equilibrium points $q_{y_{2,1}}=$ $(1,0)$ and $q_{y_{2,2}}=(-1,0)$, we obtain that they are saddle-nodes.

On the local chart $U_{1}$ system (9) becomes

$$
\begin{aligned}
& \dot{u}=u\left[3\left(v^{2}-1\right)+\frac{\sqrt{6}}{n} u\left(1+n-n v^{2}\right)\right], \\
& \dot{v}=v(3-\sqrt{6} u)\left(v^{2}-1\right) .
\end{aligned}
$$

Its equilibrium points are $p_{y_{2}, 1}=(0,0), p_{y_{2}, 2}=(\sqrt{6} n /(2(1+n)), 0)$. The equilibrium point $p_{y_{2}, 1}$ is a hyperbolic stable node with eigenvalues -3 of multiplicity two, and $p_{y_{2}, 2}$ is a hyperbolic unstable saddle with eigenvalues 3 and $-3+3 n /(1+n)$.

On the local chart $U_{2}$ system (9) writes

$$
\dot{u}=v^{2}(\sqrt{6}-3 u)-\frac{\sqrt{6}(1+n)}{n} u^{2}+3 u^{3}, \dot{v}=-\frac{\sqrt{6}}{n} u v .
$$


The $p_{y_{2,3}}=(0,0)$ is a linearly zero equilibrium point, i.e. its linear part is identically zero. Its topological index is zero by the Poincaré-Hopf theory (see Theorem 6.30 of [16] for more details).

In order to study the local phase portraits of the equilibrium point $(0,0)$ of system (12) we apply the blow-up techniques (see [17] for more details). We do a vertical blow-up by introducing the transformation $w=v / u$, then we have

$$
\begin{aligned}
& \dot{u}=u^{2}\left[-\frac{\sqrt{6}}{n}+(\sqrt{6}-3 u)\left(w^{2}-1\right)\right], \\
& \dot{w}=-u w(\sqrt{6}-3 u)\left(w^{2}-1\right) .
\end{aligned}
$$

The common factor $u$ of system (13) can be eliminated by rescaling the time $u d t=d \tau$. So we get

$$
\begin{aligned}
& u^{\prime}=u\left[-\frac{\sqrt{6}}{n}+(\sqrt{6}-3 u)\left(w^{2}-1\right)\right], \\
& w^{\prime}=-w(\sqrt{6}-3 u)\left(w^{2}-1\right),
\end{aligned}
$$

where the prime represents the derivative with respect to the time $\tau$.

Note that system (14) has three equilibrium points $p_{y_{2,4}}=(0,-1)$, $p_{y_{2}, 5}=(0,0)$ and $p_{y_{2,6}}=(0,1)$ on $u=0$. Therefore both the equilibrium points $p_{y_{2,4}}$ and $p_{y_{2,6}}$ are hyperbolic stable nodes with eigenvalues $-\sqrt{6} / n$ and $-2 \sqrt{6}$, the equilibrium point $p_{y_{2}, 5}$ is a hyperbolic unstable saddle with eigenvalues $-\sqrt{6}(1+n) / n$ and $\sqrt{6}$. The local phase portraits around these three equilibrium points are shown in Figure 4(a). Considering that there is a time rescaling $d \tau=u d t$ between systems (13) and (14), so the local phase portraits of system (13) is shown in 
Figure 4(b). Moreover, all points on the $w$-axis, i.e. $u=0$, are singularities of system (13). Thus the local phase portraits of system (12) is shown in Figure 4(c), and then the local phase portrait at the origins of $U_{2}$ and $V_{2}$ for $y_{2}=0$ can be found in Figure $4(\mathrm{~d})$.

In summary joining the previous information we obtain the global phase portraits in Figure 5 in the Poincaré disc of the plane $y_{2}=0$ restricted to the strip $-1 \leq y_{1} \leq 1$.

2.4. The invariant plane $y_{3}=0$. On this plane system (1) writes

$$
\frac{d y_{1}}{d t}=3 y_{1}\left(y_{1}^{2}-1\right), \frac{d y_{2}}{d t}=y_{2}\left(3 y_{1}^{2}-2\right)
$$

This system has three equilibrium points $O_{y_{3}, 0}=(0,0), q_{y_{3}, 1}=(1,0)$ and $q_{y_{3,2}}=(-1,0)$. Then $O_{y_{3,0}}$ is a hyperbolic stable node with eigenvalues -3 and -2 , both $q_{y_{3,1}}$ and $q_{y_{3,2}}$ are unstable hyperbolic nodes with eigenvalues 1 and 6 .

On the local chart $U_{1}$ system (15) becomes

$$
\dot{u}=u v^{2}, \dot{v}=3 v\left(v^{2}-1\right)
$$

So all the infinity of this system is filled of equilibrium points. We note that removing $v$ of system (16) doing the change of time $d \tau=v d t$, we get the system

$$
u^{\prime}=u v, v^{\prime}=3\left(v^{2}-1\right)
$$

which has no infinite equilibrium points.

On the local chart $U_{2}$ system (15) writes

$$
\dot{u}=-u v^{2}, \dot{v}=v\left(-3 u^{2}+2 v^{2}\right) .
$$




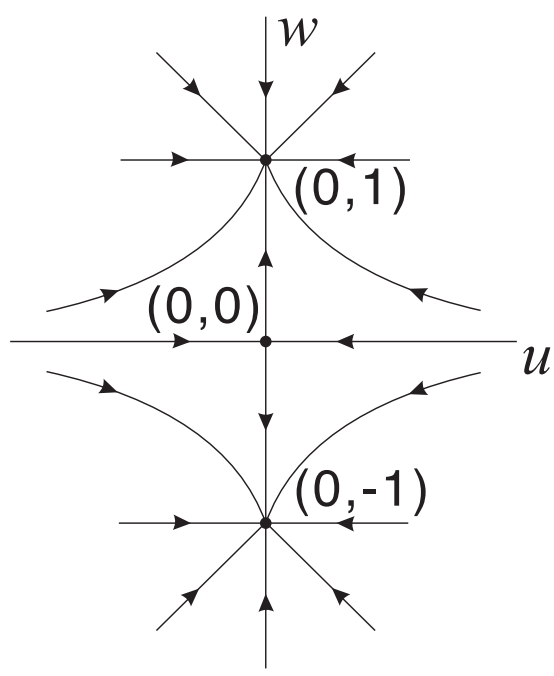

(a)

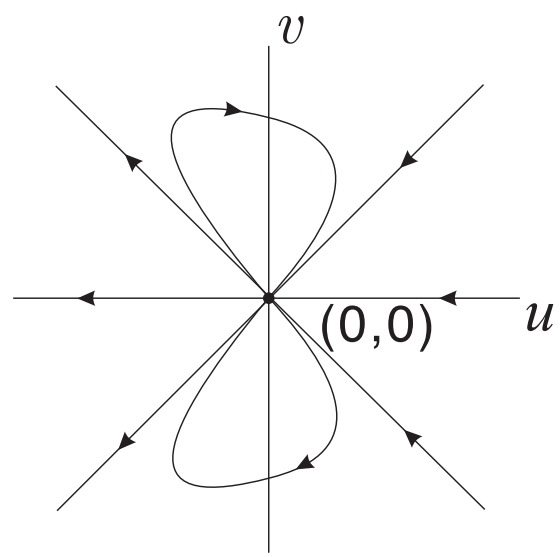

(c)

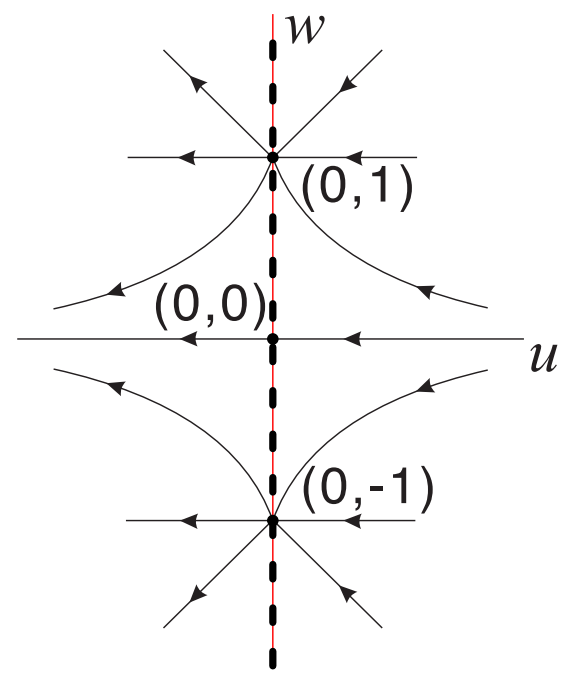

(b)

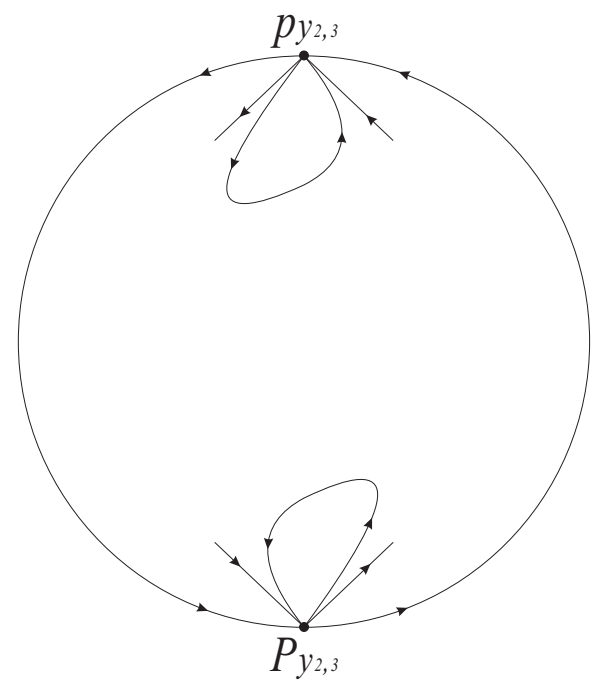

(d)

Figure 4. In (a), (b) and (c) there are the local phase portraits of the equilibrium points in system (14), (13) and (12), respectively. In (d) there is the local phase portraits at the origin of $U_{2}$ and $V_{2}$ for $y_{2}=0$.

Again doing the change of variable $d \tau=v d t$ system (18) becomes

$$
u^{\prime}=-u v, v^{\prime}=-3 u^{2}+2 v^{2} .
$$



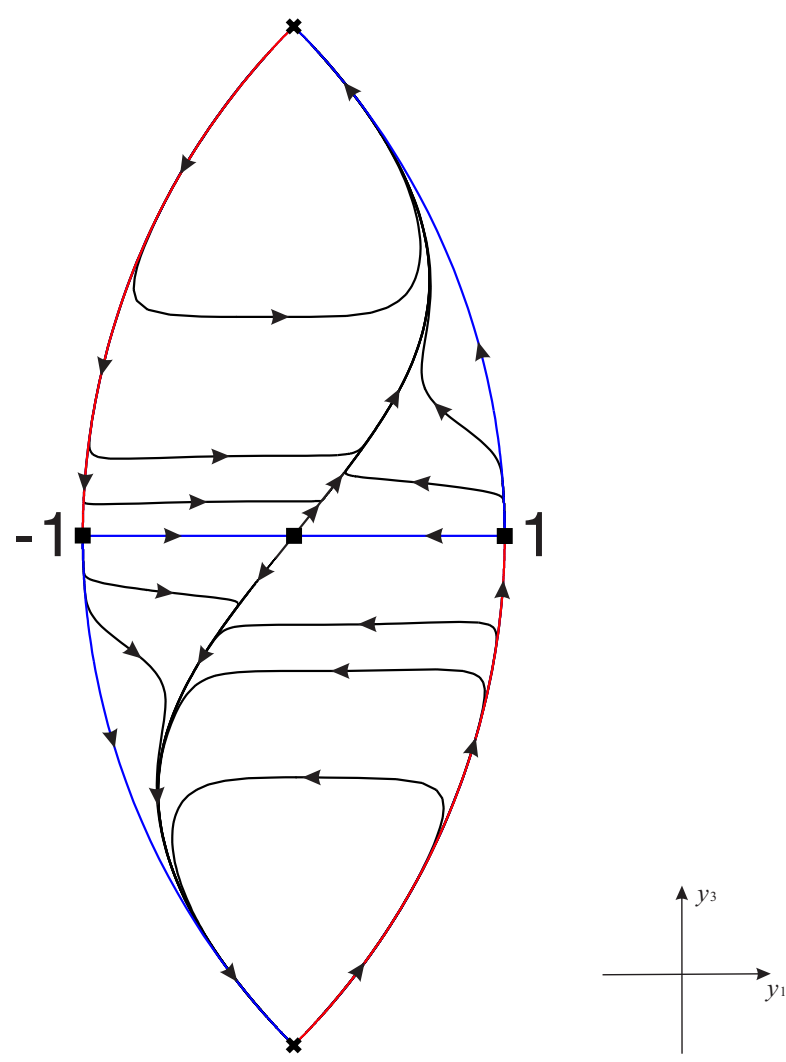

FiguRE 5. The phase portraits of the invariant plane $y_{2}=0$ restricted to $-1 \leq y_{1} \leq 1$.

The $p_{y_{3,1}}=(0,0)$ is also a linearly zero equilibrium point of system (19). Applying again the blow-up techniques of [17] to $p_{y_{3,1}}$, then we obtain

$$
u^{\prime}=-u^{2} w, w^{\prime}=3 u\left(w^{2}-1\right),
$$

after doing the change of variable $w=v / u$. Rescaling the time of system (20) we get

$$
\dot{u}=-u w, \dot{w}=3\left(w^{2}-1\right) \text {. }
$$


Hence system (21) has two equilibrium points $p_{y_{3,2}}=(0,-1)$ and $p_{y_{3,3}}=(0,1)$ on $u=0$. Therefore both the equilibrium points $p_{y_{3,2}}$ and $p_{y_{3,3}}$ are hyperbolic unstable saddles with eigenvalues $1,-6$ and $-1,6$, respectively. The local phase portraits around these two equilibrium points is shown in Figure 6(a). Considering that there is time $d \tau=u d t$ to rescale between systems (20) and (21), the local phase portraits of system (20) is shown in Figure 6(b). In addition, all points on the $w$-axis, i.e. $u=0$ are the singularities of the system (20). Therefore the local phase portraits of system (19) is shown in Figure 6(c), and then the local phase portraits at the origins of $U_{2}$ and $V_{2}$ of $y_{3}=0$ can be found in Figure 6(d).

In conclusion from the previous information and taking into account that the straight lines $y_{1}=0$ and $y_{2}=0$ are invariant under the flow of system (15), we obtain the global phase portraits restricted to the band $-1 \leq y_{1} \leq 1$ in Figure 7 in the Poincare disc of the plane $y_{3}=0$.

3. Phase portraits inside the Poincaré Ball Restricted to $-1 \leq y_{1} \leq 1$

We divide the Poincaré ball restricted to $-1 \leq y_{1} \leq 1$ into four regions:

$$
\begin{array}{ll}
R_{1}: y_{2} \leq 0, y_{3} \geq 0 . & R_{2}: y_{2} \leq 0, y_{3} \leq 0 \\
R_{3}: y_{2} \geq 0, y_{3} \geq 0 . & R_{4}: y_{2} \geq 0, y_{3} \leq 0
\end{array}
$$

Since system (1) is invariant under the symmetry with respect to the $y_{2}$-axis, i.e. $\left(y_{1}, y_{2}, y_{3}\right)=\left(-y_{1}, y_{2},-y_{3}\right)$, we only need to study the phase portraits in the regions $R_{1}$ and $R_{2}$. 


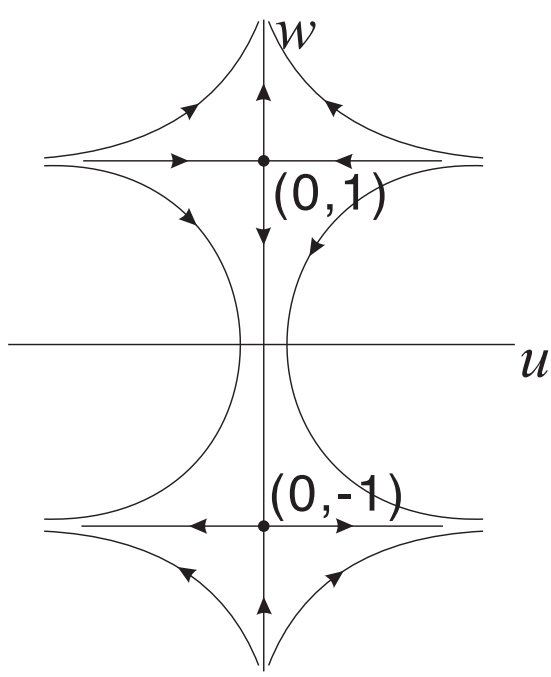

(a)

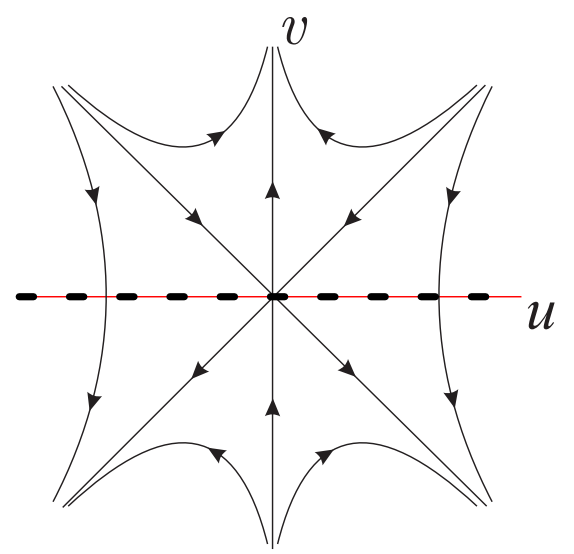

(c)

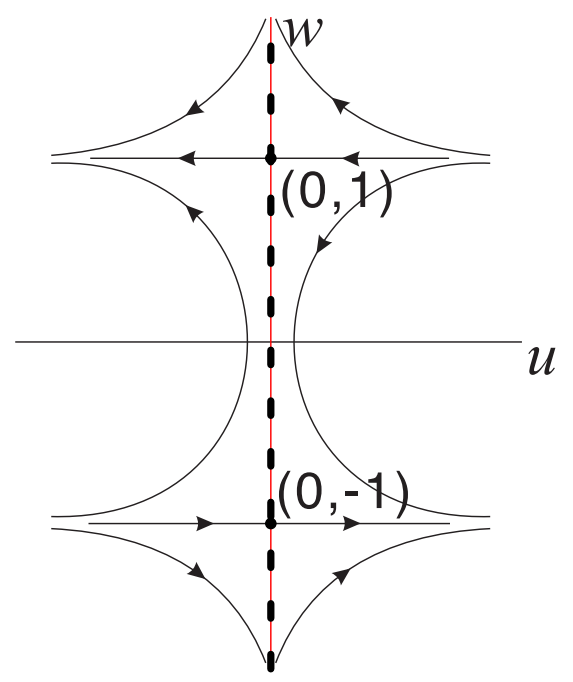

(b)

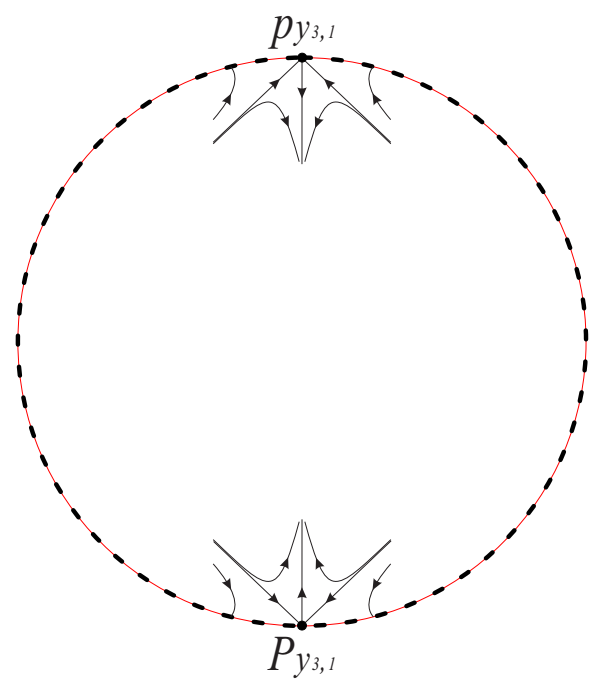

(d)

FiguRE 6. In (a), (b) and (c) there are the local phase portraits of the equilibrium points in system (21), (20) and (19), respectively. In (d) there is the local phase portraits at the origins of $U_{2}$ and $V_{2}$ for $y_{3}=0$.

Putting together the phase portraits of the invariant planes $y_{1}= \pm 1$, $y_{2}=0$ and $y_{3}=0$ we obtain the phase portraits in the boundary of 

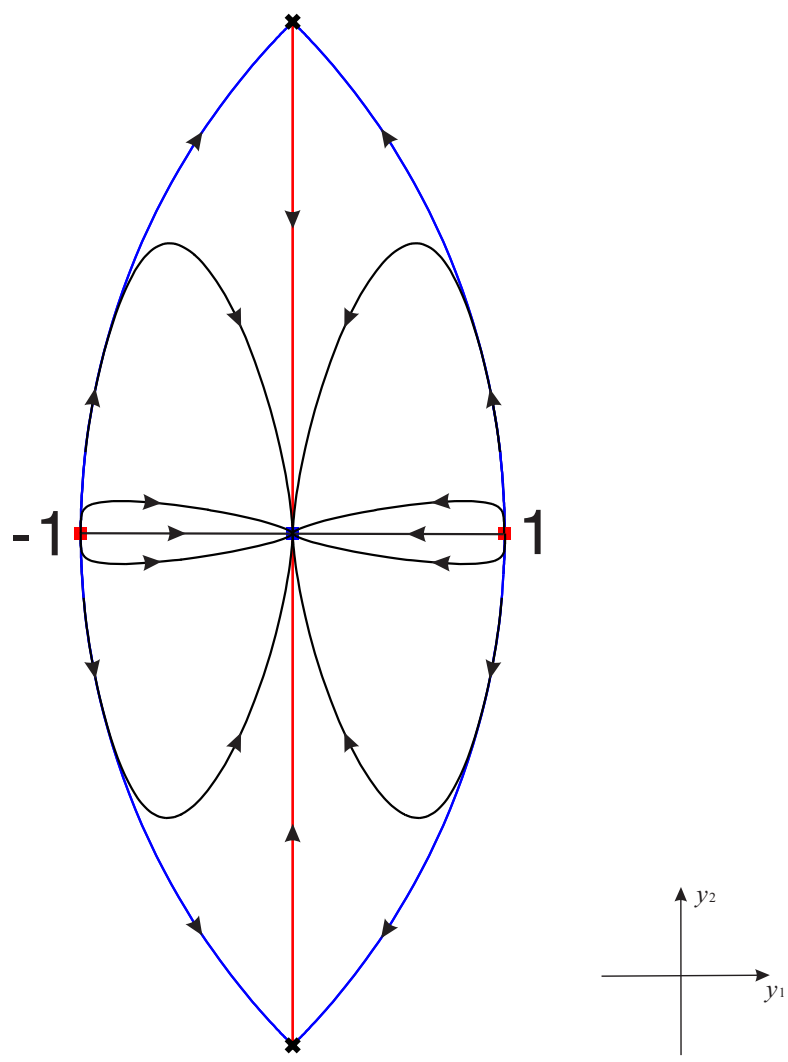

FiguRE 7 . The phase portrait of the invariant plane $y_{3}=0$ restricted to $-1 \leq y_{1} \leq 1$.

the regions $R_{1}$ and $R_{2}$ in Figures 8 and 9 , respectively. The threedimensional cartesian coordinate system in this paper is defined as follows: we consider the $y_{1} y_{2}$-plane as the horizontal plane in $\mathbb{R}^{3}$, in which the direction of the $y_{2}$-axis is horizontally to the right. If the $y_{2}$-axis is rotated 90 degrees counterclockwise we get the $y_{1}$-axis. The $y_{3}$-axis is vertically upward, then $y_{1} y_{2} y_{3}$ constitutes a three dimensional left-handed cartesian coordinate system.

These five planes divide the regions $R_{1}$ and $R_{2}$ into twelve different subregions $G_{i}, i=(1,2, \cdots, 12)$ (see Figures 10 and 11 for more details). 

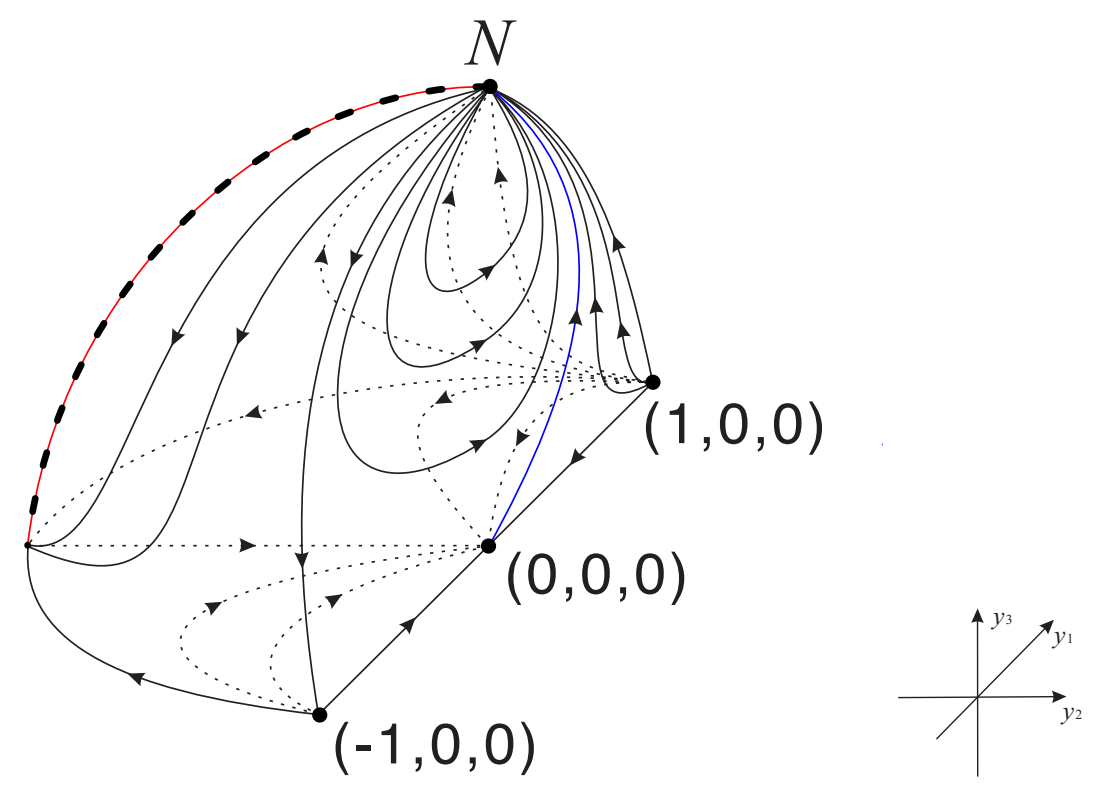

Figure 8. Phase portrait in the boundary of the region $R_{1}$. $N$ denotes the North Pole of the Poincaré ball.

Note that the original system (1) admits three finite equilibrium points $(-1,0,0),(0,0,0)$ and $(1,0,0)$. The dynamical behavior of the system inside the regions $R_{1}$ and $R_{2}$ depends on the behavior of the flow in the following five planes

$$
y_{1}=a y_{3}, y_{1}= \pm a, y_{1}=0, y_{3}=0
$$

where $a=\sqrt{6} / 3$.

4. Dynamics in the interior of the Regions $R_{1}$ And $R_{2}$

In Table 1 we describe the behavior of $\dot{y_{1}}, \dot{y_{2}}$ and $\dot{y_{3}}$ in the twelve subregions $G_{i}$ for $i=1,2, \cdots, 12$. From this table we get that the orbits from the subregion $G_{1}$ must go to the equilibrium point $N$, this 

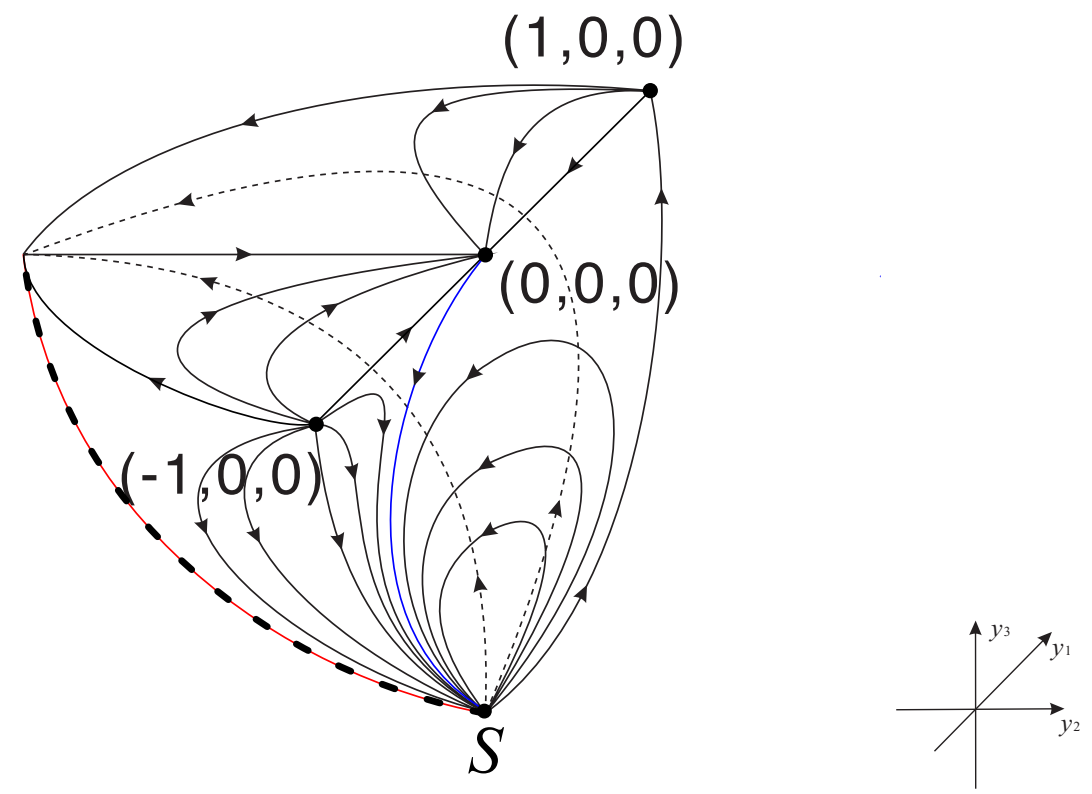

Figure 9. Phase portrait in the boundary of the region $R_{2}$. S denotes the South Pole of the Poincaré ball.
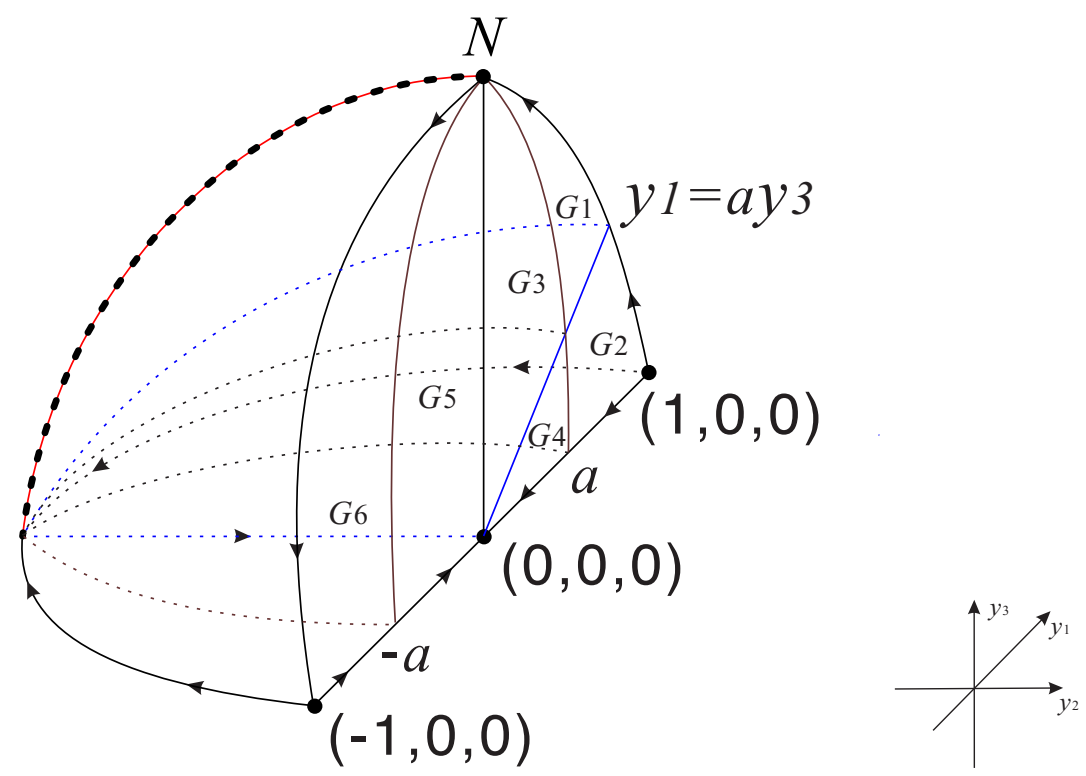

Figure 10. The six subregions of $R_{1}$ in the Poincaré ball. 

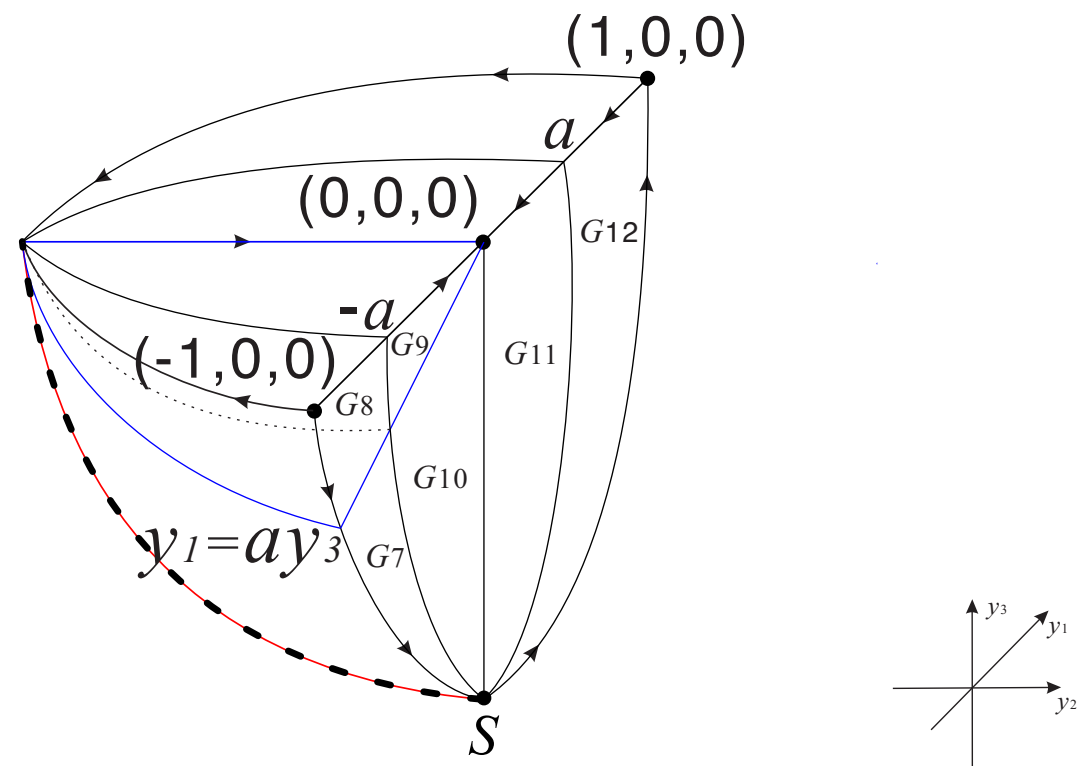

Figure 11. The six subregions of $R_{2}$ in the Poincaré ball.

is represented as follows

$$
G_{1} \rightarrow N
$$

In a similar way and taking into account that $y_{2}=0$ and $y_{3}=0$ are invariant planes, we obtain that

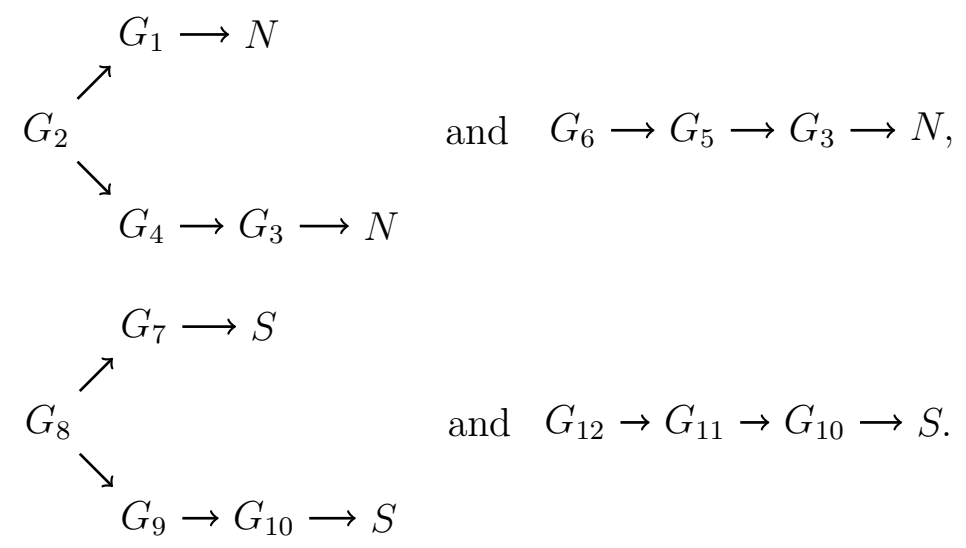


TABLE 1. Dynamical behavior in twelve different subregions

\begin{tabular}{ccc}
\hline Subregions & Corresponding Region & Increase or decrease \\
\hline$G_{1}$ & $y_{1}<y_{3}, \sqrt{6} / 3<y_{1}<1, y_{3}>0$ & $\dot{y_{1}}>0, \dot{y_{2}}<0, \dot{y_{3}}>0$ \\
\hline$G_{2}$ & $y_{1}>y_{3}, \sqrt{6} / 3<y_{1}<1, y_{3}>0$ & $\dot{y_{1}}<0, \dot{y_{2}}<0, \dot{y_{3}}>0$ \\
\hline$G_{3}$ & $y_{1}<y_{3}, 0<y_{1}<\sqrt{6} / 3, y_{3}>0$ & $\dot{y_{1}}>0, \dot{y_{2}}>0, \dot{y_{3}}>0$ \\
\hline$G_{4}$ & $y_{1}>y_{3}, 0<y_{1}<\sqrt{6} / 3, y_{3}>0$ & $\dot{y_{1}}<0, \dot{y_{2}}>0, \dot{y_{3}}>0$ \\
\hline$G_{5}$ & $y_{1}<y_{3},-\sqrt{6} / 3<y_{1}<0, y_{3}>0$ & $\dot{y_{1}}>0, \dot{y_{2}}>0, \dot{y_{3}}<0$ \\
\hline$G_{6}$ & $y_{1}<y_{3},-1<y_{1}<-\sqrt{6} / 3, y_{3}>0$ & $\dot{y_{1}}>0, \dot{y_{2}}>0, \dot{y_{3}}<0$ \\
\hline$G_{7}$ & $y_{1}>y_{3},-1<y_{1}<-\sqrt{6} / 3, y_{3}<0$ & $\dot{y_{1}}<0, \dot{y_{2}}<0, \dot{y_{3}}<0$ \\
\hline$G_{8}$ & $y_{1}<y_{3},-1<y_{1}<-\sqrt{6} / 3, y_{3}<0$ & $\dot{y_{1}}>0, \dot{y_{2}}<0, \dot{y_{3}}<0$ \\
\hline$G_{9}$ & $y_{1}<y_{3},-\sqrt{6} / 3<y_{1}<0, y_{3}<0$ & $\dot{y_{1}}>0, \dot{y_{2}}>0, \dot{y_{3}}<0$ \\
\hline$G_{10}$ & $y_{1}>y_{3},-\sqrt{6} / 3<y_{1}<0, y_{3}<0$ & $\dot{y_{1}}<0, \dot{y_{2}}>0, \dot{y_{3}}<0$ \\
\hline$G_{11}$ & $y_{1}>y_{3}, 0<y_{1}<\sqrt{6} / 3, y_{3}<0$ & $\dot{y_{1}}<0, \dot{y_{2}}>0, \dot{y_{3}}>0$ \\
\hline$G_{12}$ & $y_{1}>y_{3}, \sqrt{6} / 3<y_{1}<1, y_{3}<0$ & $\dot{y_{1}}<0, \dot{y_{2}}<0, \dot{y_{3}}>0$ \\
\hline
\end{tabular}

If we define the subregions

$$
\begin{aligned}
& R_{1+}=\left\{\left(y_{1}, y_{2}, y_{3}\right) \in R_{1}: 0<y_{1}<1\right\}, \\
& R_{1-}=\left\{\left(y_{1}, y_{2}, y_{3}\right) \in R_{1}:-1<y_{1}<0\right\}, \\
& R_{2+}=\left\{\left(y_{1}, y_{2}, y_{3}\right) \in R_{2}: 0<y_{1}<1\right\}, \\
& R_{2-}=\left\{\left(y_{1}, y_{2}, y_{3}\right) \in R_{2}:-1<y_{1}<0\right\},
\end{aligned}
$$

the orbits obtained from Table 1 says that the orbits of system (1) contained in the region $R_{1+}$ have $\alpha$-limit at the equilibrium point $(1,0,0)$ and $\omega$-limit at the infinite equilibrium point $N$; the orbits of system (1) contained in the region $R_{1-}$ have $\alpha$-limit and $\omega$-limit at the infinite equilibrium point $N$; the orbits of system (1) contained in the region $R_{2+}$ have $\alpha$-limit and $\omega$-limit at the infinite equilibrium point $S$; finally the orbits of system (1) contained in the region $R_{2-}$ have $\alpha$-limit at 
the equilibrium point $(-1,0,0)$ and $\omega$-limit at the infinite equilibrium point $S$.

This completes the description of all the qualitative dynamics of the system (1).

\section{ACKNOWLEDGMENTS}

The first author gratefully acknowledges the support of the National Natural Science Foundation of China (NSFC) through grant Nos.11672259 and 11571301, the China Scholarship Council through grant No.201908320086, the Ministry of Land and Resources Research of China in the Public Interest through grant No.201411007.

The second author gratefully acknowledges the support of the Ministerio de Economía, Industria y Competitividad, Agencia Estatal de Investigación grants MTM2016-77278-P (FEDER) and MDM-20140445, the Agència de Gestió d'Ajuts Universitaris i de Recerca grant 2017SGR1617, and the H2020 European Research Council grant MSCARISE-2017-777911.

\section{REFERENCES}

[1] P. Hořava, Quantum gravity at a Lifshitz point, Physical Review D 79, 084008, 2009.

[2] S. Carloni, E. Elizalde and P.J. Silva, An analysis of the phase space of Hořava-Lifshitz cosmologies. In: S.D. Odintsov, D. Sáez-Gómez and S. XambóDescamps (eds) Cosmology, Quantum Vacuum and Zeta Functions, Springer Proceedings in Physics 137, 139-148, Springer-Verlag, Berlin, 2011.

[3] M. Li and Y. Pang, A trouble with Hořava-Lifshitz gravity, Journal of High Energy Physics 08, 015, 2009. 
[4] O. Luongo, M. Muccino and H. Quevedo, Kinematic and statistical inconsistencies of Hořava-Lifshitz cosmology, Physics of the Dark Universe 25, 100313, 2019.

[5] N.A. Nilsson and E. Czuchry, Hořava-Lifshitz cosmology in light of new data, Physics of the Dark Universe 23, 100253, 2019.

[6] S. Mukohyama, Hořava-Lifshitz cosmology: a review, Classical and Quantum Gravity 27, 223101, 2010.

[7] T.P. Sotiriou, Hořava-Lifshitz gravity: a status report, Journal of Physics: Conference Series 283, 012034, 2011.

[8] G. Leon, and E.N. Saridakis, Phase-space analysis of Hořava-Lifshitz cosmology, Journal of Cosmology and Astroparticle Physics 2009, 006, 2009.

[9] G. Leon, and A. Paliathanasis, Extended phase-space analysis of the HořavaLifshitz cosmology, arXiv preprint arXiv: 1902.09961v2, 2019.

[10] E.N. Saridakis, Aspects of Hořava-Lifshitz cosmology, International Journal of Modern Physics D 20 (08), 1485-1504, 2011.

[11] B. Chen, On Hořava-Lifshitz cosmology, Chinese Physics C 35 (5), 429-435, 2011.

[12] S. Lepe, J. Saavedra, On Hořava-Lifshitz cosmology, Astrophysics and Space Science 350, 839-843, 2014.

[13] E.M.C. Abreu, A.C.R. Mendes, G. Oliveira-Neto et al., Hořava-Lifshitz cosmological models with noncommutative phase space variables, General Relativity and Gravitation 51, 95, 2019.

[14] A. Paliathanasis, and G. Leon, Cosmological solutions in Hořava-Lifshitz gravity, arXiv preprint arXiv: 1903.10821, 2019.

[15] M.R. Setare and D. Momeni, Plane symmetric solutions in Hořava-Lifshitz theory, International Journal of Modern Physics D 19(13), 2079-2094, 2010.

[16] F. Dumortier, J. Llibre, and J.C. Artés, Qualitative theory of planar differential systems, Springer-Verlag, Berlin, 2006.

[17] M.J. Álvarez, A. Ferragut, and X. Jarque, A survey on the blow up technique, International Journal of Bifurcation and Chaos 21(11), 3103-3118, 2011. 
${ }^{1}$ School of Mathematical Science, Yangzhou University, Yangzhou 225002, CHINA

E-mail: gaofabao@sina.com (Fabao Gao)

2 Departament de Matemàtiques, Universitat Autònoma de Barcelona, Bellaterra 08193, Barcelona, Catalonia, Spain

E-mail: jllibre@mat.uab.cat (Jaume Llibre) 